\section{TA 7.4 Phase-state Low Electron-number Drive Random Access Memory (PLEDM)}

Kazuo Nakazato, Kiyoo Itoh'1, Haroon Ahmed², Hiroshi Mizuta, Teruaki Kisu³, Masataka Kato ${ }^{4}$, Takeshi Sakata ${ }^{1}$

Hitachi Cambridge Lab Hitachi Europe Ltd., Cambridge, U.K.

${ }^{1}$ Central Research Labs Hitachi Ltd. Tokyo, Japan

${ }^{2}$ Cavendish Lab Cambridge Univ., Cambridge, U.K.

${ }^{3}$ Hitachi ULSI Systems Co. Tokyo, Japan

${ }^{4}$ Semiconductor Division, Hitachi Ltd. Tokyo, Japan

Dynamic random access memories (DRAMs) based on a transistor/capacitor cell are used as main memories in computers because of their high capacity and high speed. Since there is no gain in the present DRAM cell, it requires a large cell-capacitor to produce an adequate sense signal. In each new memory generation, structure and fabrication have become more complicated to maintain a large capacitor while miniaturizing the cell.

This phase-statelow el ectron-number drivememory (PLEDM) gain cell uses a stacked tunnel transistor (PLEDTR) [1]. Since this cell has gain, a largecapacitor is not necessary. Thecell sizeis $5 F^{2}$ where $F$ is the minimum feature size. Its read and write times are simulated as $20 \mathrm{~ns}$ and $5 \mathrm{~ns}$, respectively. In principle, it is possible with PLEDM to havea retention timelonger than 10 years, enabling a non-volatile memory to be realised [2].

PLEDTR is a vertical fully depleted double-gateSOI-MOSFET with barriers in the channel region (Figure 7.4.1). Gate voltage modulates the internal potential in the intrinsic silicon region and the central shutter barrier or barriers (CSB) move up and down energetically following the internal potential. The CSB reduce OFF current substantially, while keeping device ON current high. The device may be regarded as a three-terminal version of the heterostructure hot-electron diode $\left(H^{2} E D\right)$ based on transition from a tunneling current to a thermoionic emision current at a semiconductor heterojunction [3]. The role of the source and drain barriers is (1) toincreasetheweighting of thehigh-energy part of theinjected electrons (2) toact as impurity diffusion barriers which ensurea low impurity level within thechannel, and (3) to reduceleakage current such as gate-induced drain leakage current at the drain side.

PLEDTRs with triple tunnel barriers are fabricated on silicon dioxide using a standard silicon process, as shown in Figure 7.4.2. All transistor regions, source, drain, channel, and gate, are polycrystalline-silicon films. The thin tunnel junctions are formed by thermal nitridation of silicon at $900^{\circ} \mathrm{C}$. Sourceand drain regions are heavily phosphorous doped to $2 \times 10^{20} \mathrm{~cm}^{-3}$, while the channel region is maintained at impurity level $<10^{17} \mathrm{~cm}^{-3}$ as confirmed by SIMS analysis. The gate is boron doped to $5 \times 10^{19} \mathrm{~cm}^{-3}$. The channel length is $60 \mathrm{~nm}$ and the gate insulator is formed by $6 \mathrm{~nm}$ of silicon dioxide. The gateseparation width is $45 \mathrm{~nm}$, and the gatewidth, perpendicular dimension to the plane of Figure 7.4.2a, is $0.4 \mu \mathrm{m}$.

Drain current measurements shown in Figure 7.4.2b demonstrate $<\mathrm{lf}$ A leakage. The threshold voltage is $\sim 1 \mathrm{~V}$, and sub-threshold voltage slope $S$ is $96 \mathrm{mV} /$ decade. This sub-threshold voltage slope is explained well by thescaling theory for a double-gateSOI MOSFET [4], and the same slope $S$ is obtained for PLEDTR with the $0.2 \mu \mathrm{m}$ gate separation and 350nm channel length [5].

PLEDTR enables construction of high-density memory because each memory cell occupies the area of one transistor, as shown in Figure 7.4.3. A PLEDTR is stacked onto the gate of a conventional MOSFET with a built-in coupling capacitor torealizea memory cell. High-speed write is possible by transferring electrons from the top electrode (data line) onto the memory node through the ON-state PLEDTR. Since the OFF-state PLEDTR effectively confines electrons, the stored information can be kept for a long time without refresh. Since the information is read via thecurrent in a MOSFET, this cell has gain and a large $\mathrm{S} / \mathrm{N}$ ratio.

Storage, read, and writecycles areall controlled by voltage $V_{w}$ on the word line, $V_{w}^{(S)}(-2 \mathrm{~V}), V_{w}^{(R)}(0.5 \mathrm{~V})$, and $V_{w}{ }^{(W)}(3 \mathrm{~V})$. (Figure 7.4.4) The generation of negativeword line voltageis described in reference[6]. In the storage cycle the built-in coupling capacitor $C_{C}$ causes the memory node voltage $V_{N}$ to be lower than the threshold voltage $V_{t h}$ of the sense MOSFET. I n the read cycle $V_{N}$ becomes higher than $V_{t h}$ when thememory stateis high and lower than $V_{t h}$ when thememory state is low. In the write cycle the PLEDTR is opened, and $V_{N}$ becomes the data line voltage, 1.5V for the high memory state and OV for the low memory state. Figures 7.4.4(b) and (c) show the results of mixed-level device and circuit simulation of two memory cells using $0.13 \mu \mathrm{m}$ rules. A sequence of writing high state $(\mathrm{WH})$, storage $(S)$, read $(R)$, refresh $(r), S, R$, writing low state $(W L), S, R$, $r, S, R$, with 10ns/20ns/5ns storage (pre-charge) / read/write time is simulated. $V_{D D}=1.5 \mathrm{~V} . V_{w 2}$ is kept at $-2 \mathrm{~V}$ (unselected) after writing high or low state. The refresh inverts the memory state. The inverting cell concept is described in reference [7]. Although the drain-source current in the ON state of a PLEDTR is $\sim 1 \mu \mathrm{A}$, write is fast because of the reduced stored charge, which is determined by the gate capacitance of the sense MOSFET and estimated as 0.2 to $0.3 \mathrm{fC}$. On the other hand a high ON current is available from the sense MOSFET to drive the data-line capacitance, $200 \mathrm{fF}$ in this simulation.

The memory node voltage $V_{N}$ in the storage and read cycles is calculated as a function of the coupling capacitance $C_{C}$ in Figure 7.4.5. $V_{H}^{(R)}$ and $V_{L}^{(R)}$ are in the read cycle in high and low memory states, respectively. $V_{H}^{(S)}$ and $V_{L}{ }_{L}^{(S)}$ are in the storage cycle. The voltage difference on the memory node between high and low memory states, $V_{H}-V_{L}$, can be larger than the writing voltage difference, $1.5 \mathrm{~V}$ in this case, because of the change of memory node capacitance between inversion and depletion states of the sense MOSFET. Random read access in a cell array is possible when $V_{\text {th }}$ is set in the hatched area, for example, between $0.5 \mathrm{~V}$ and $-0.5 \mathrm{~V}$ at $0.04 f \mathrm{~F}$ coupling capacitance. This coupling capacitance can be realized for a $50 \mathrm{~nm}$ thick memory node $\left(t_{N}\right)$, without needing to form an additional capacitor.

The $5 F^{2}$ memory cell layout is shown in Figure 7.4.6a. Theschematic of memory device is shown in Figure 7.4.6b. The refresh circuit consists of one transistor per column with the same pitch as the memory cell, without sense amplifiers, so the cell area occupancy ratio increases substantially. An experimental memory cell array is in fabrication.

\section{References:}

[1] Nakazato, K., P.] . A. Piotrowicz, D. G. Hasko, H. Ahmed, K. I toh, “PLED - Planar Localised Electron Devices", IEDM, pp.179-182, 1997.

[2] Mizuta, H., K. Nakazato, P. J . A. Piotrowicz K. Itoh, T. Teshima, K. Yamaguchi, T. Shimada, "Normally-off PLED (Planar Localised Electron Device) for non-volatile memory", VLSI Symposium, pp. 128-129, 1998.

[3] Hess, K., T. K. Higman, M. A. Emanuel, J . J . Coleman, "New ultrafast switching mechanism in semiconductor heterostructures", J . Appl. Phys. 60, pp. 3775-3777, 1986

[4] Suzuki, K., T. Tanaka, Y. Tosaka, H. Horie, Y. Arimoto, "Scaling theory for double-gate SOI MOSFET's", IEEE Trans. Electron Dev., ED-40, pp. 23262329, 1993.

[5] Kisu, T., K. Nakazato, "Silicon Stacked Transistor with Source and Drain Tunnel Barriers", ESSDERC, pp. 532-535, 1999.

[6] Tanaka, H., M. Aoki, T. Sakata, S. Kimura, N. Sakashita, H. Hidaka, T. Tachibana, K. Kimura, "A PreciseOn-Chip Voltage Generator for a Gigascale DRAM with a Negative Word-Line Scheme", IEEE J SSC 34, pp.1084-1090, 1999.

[7] Martino, W., B. F. Croxon, "The Inverting Cell Concept for MOS Dynamic RAMS", IEEE ISSCC, pp. 12-13, 1972. 


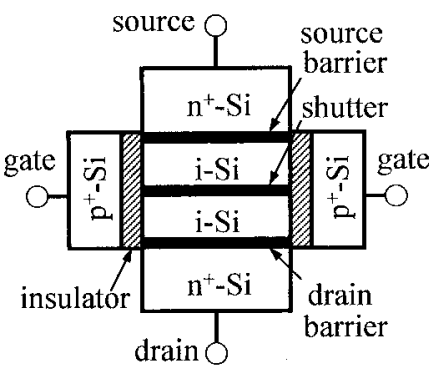

(a)

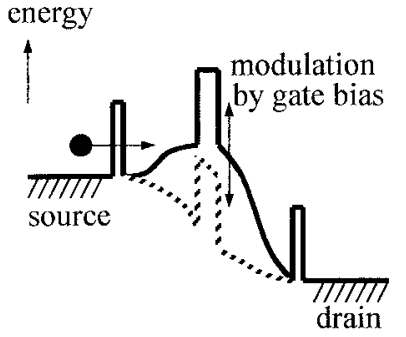

(b)
Figure 7.4.1: PLEDTR. (a) Schematic cross-section, (b) conduction-band energy diagram.

silicon oxide data line

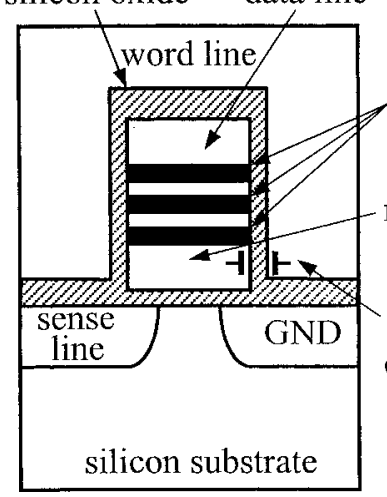

(a)

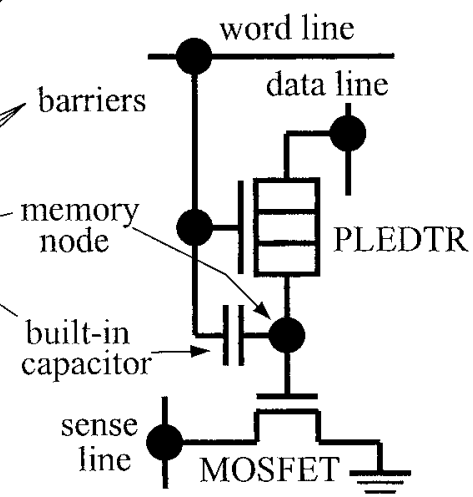

(b)
Figure 7.4.3: PLEDM cell (a) cross-section,

(b) equivalent circuit diagram.

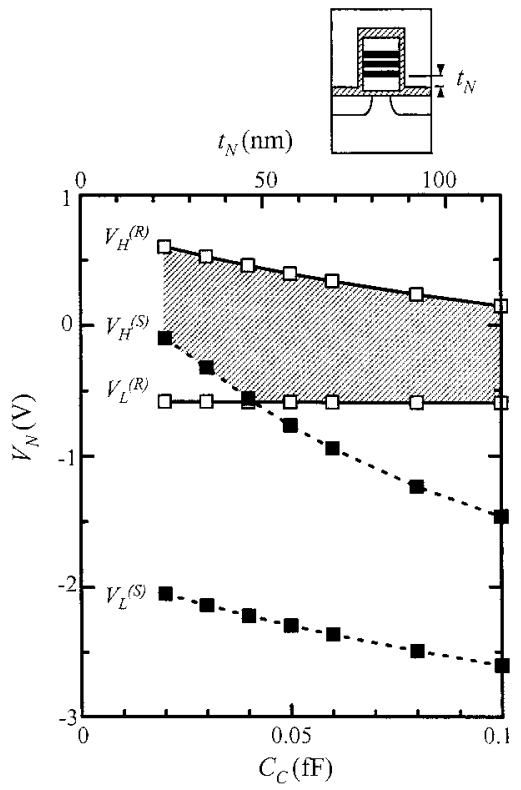

Figure 7.4.5: Simulated memory node voltages.
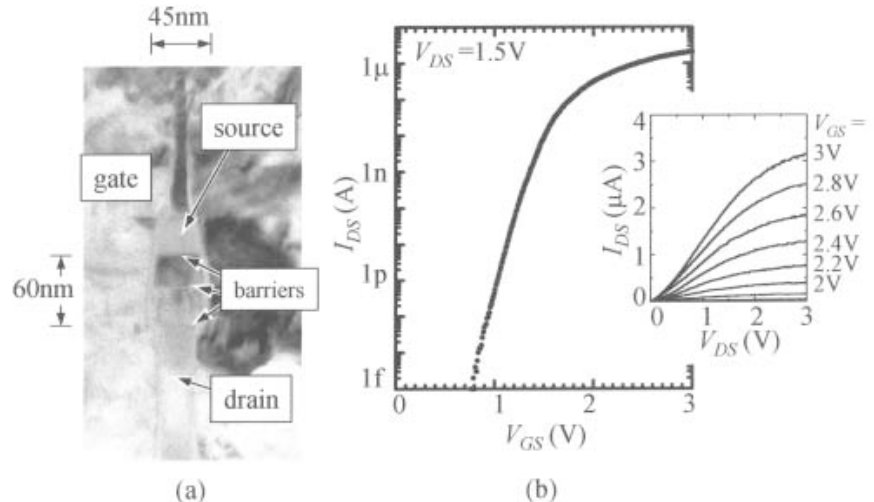

(b)

Figure 7.4.2: Fabricated PLEDTR. (a) Transmission electron micrograph, (b) measured drain current.
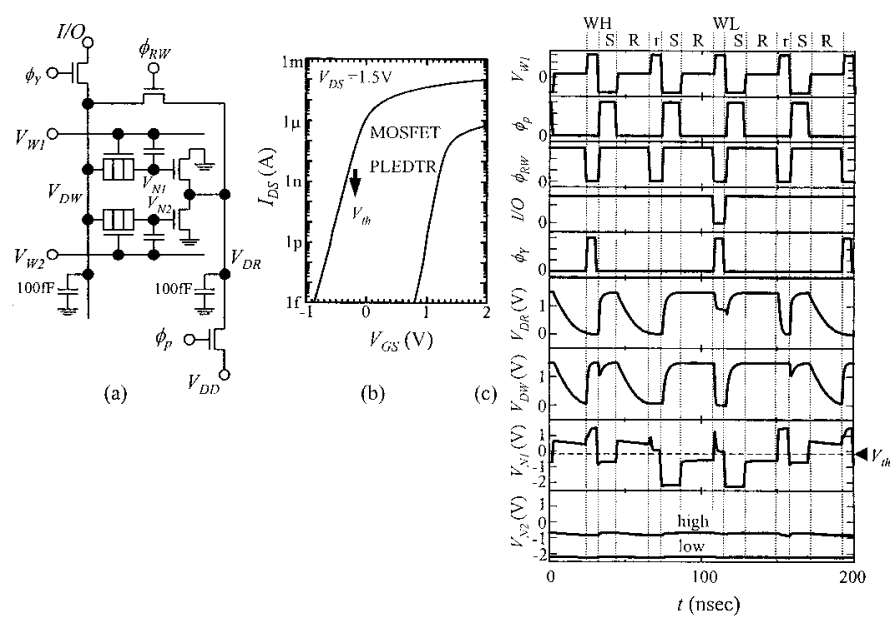

Figure 7.4.4: Simulation of memory cells.

(a) circuit diagram,

(b) transistor characteristics, (c) waveforms.

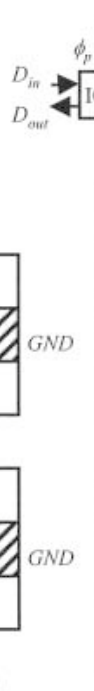

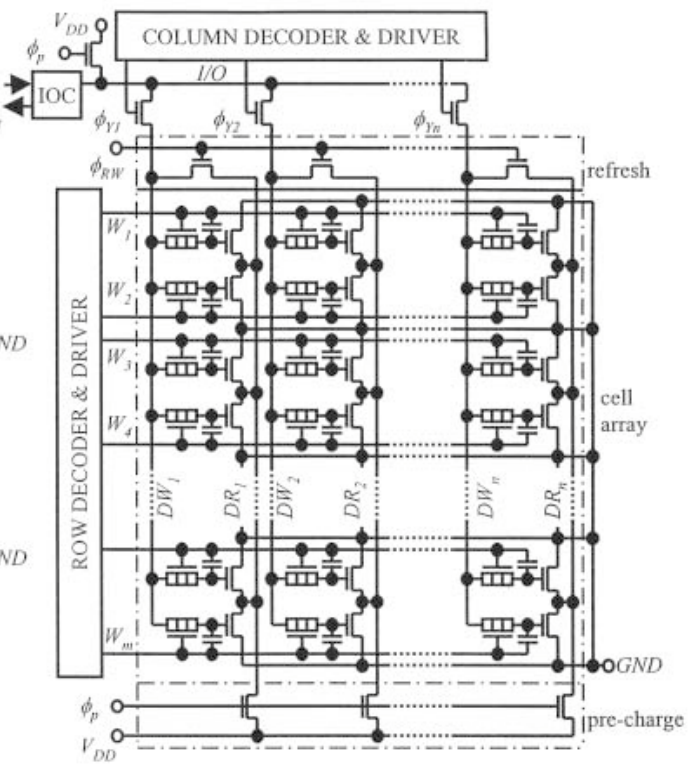

(b) (a)

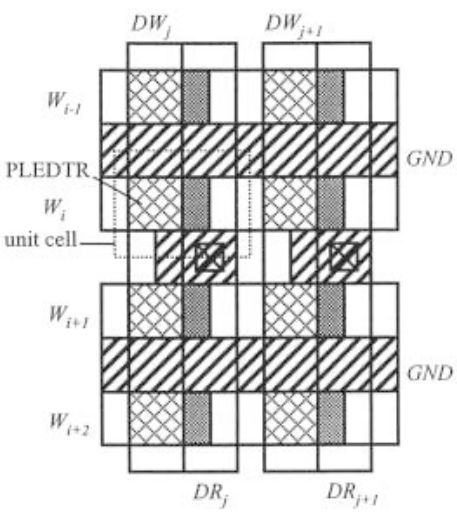

Figure 7.4.6: Memory device (a) cell layout, (b) schematic diagram. 


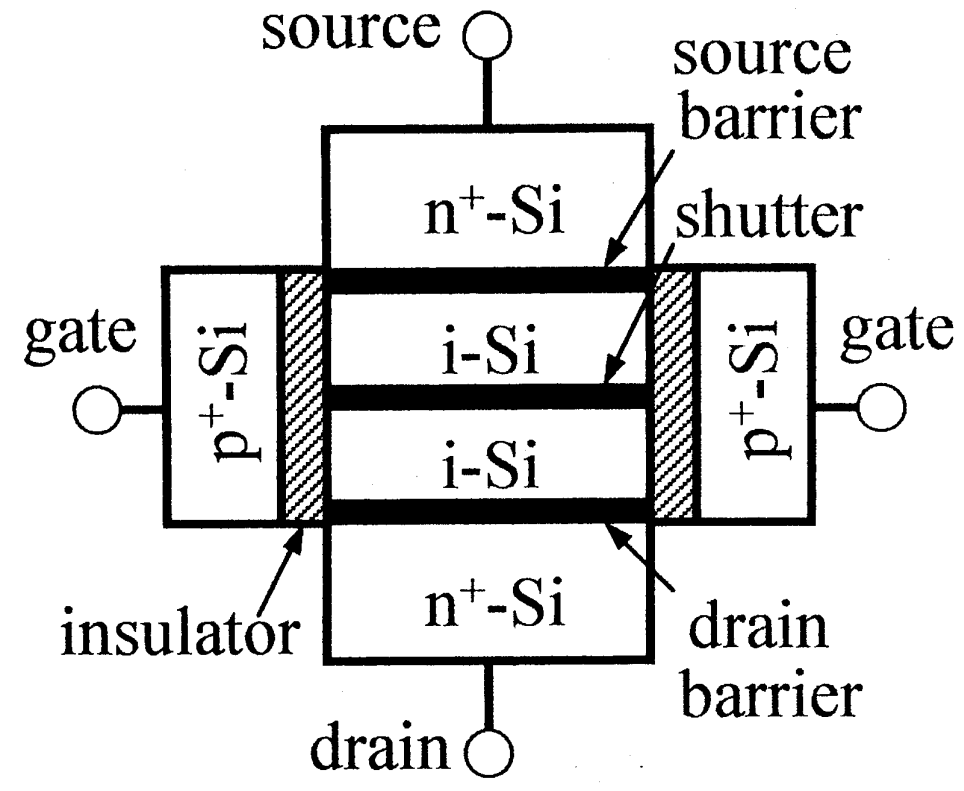

(a)

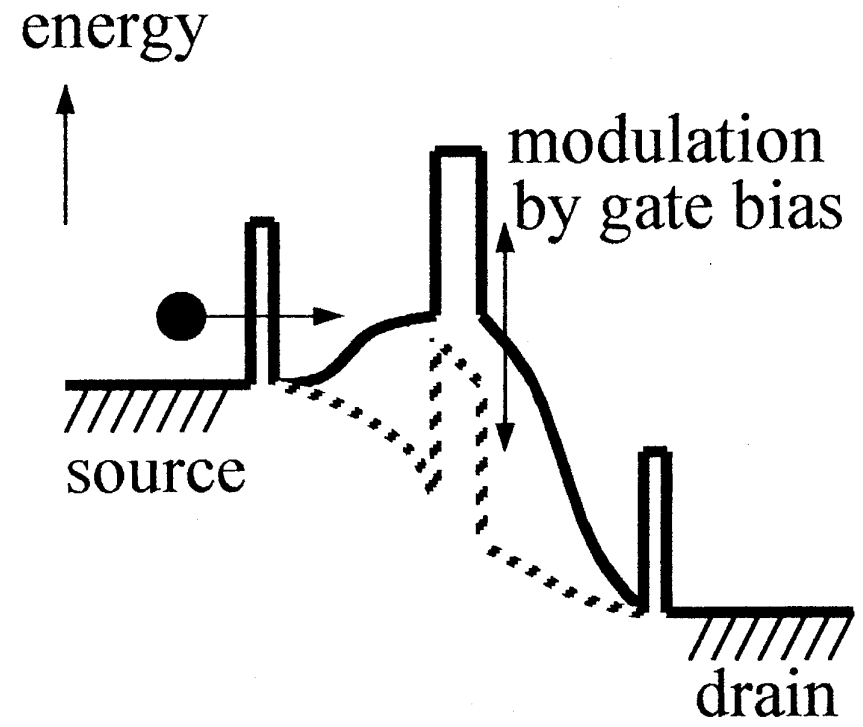

(b)

Figure 7.4.1: PLEDTR. (a) Schematic cross-section, (b) conduction-band energy diagram. 


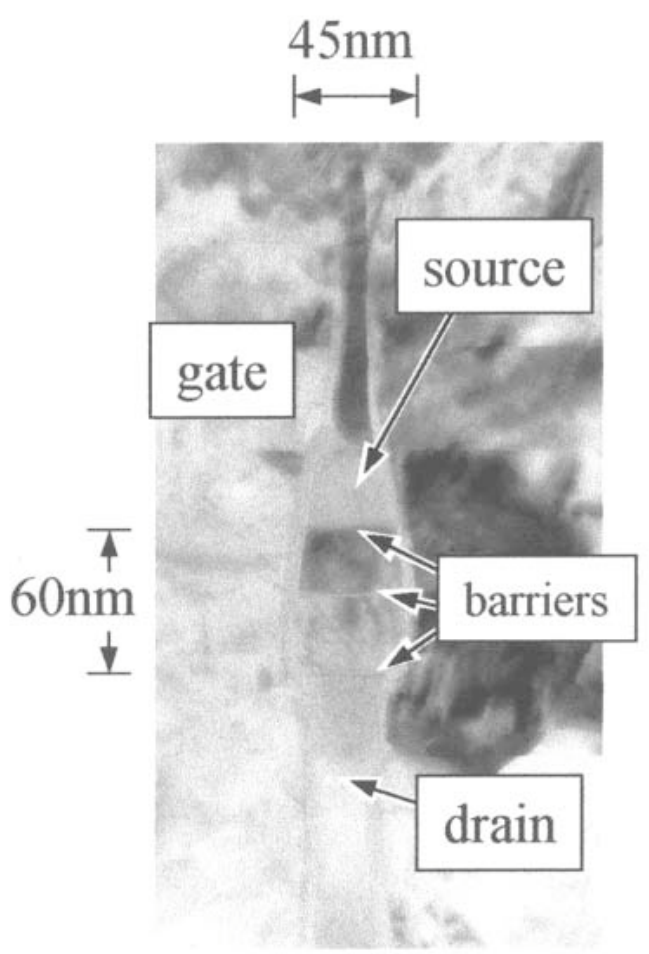

(a)

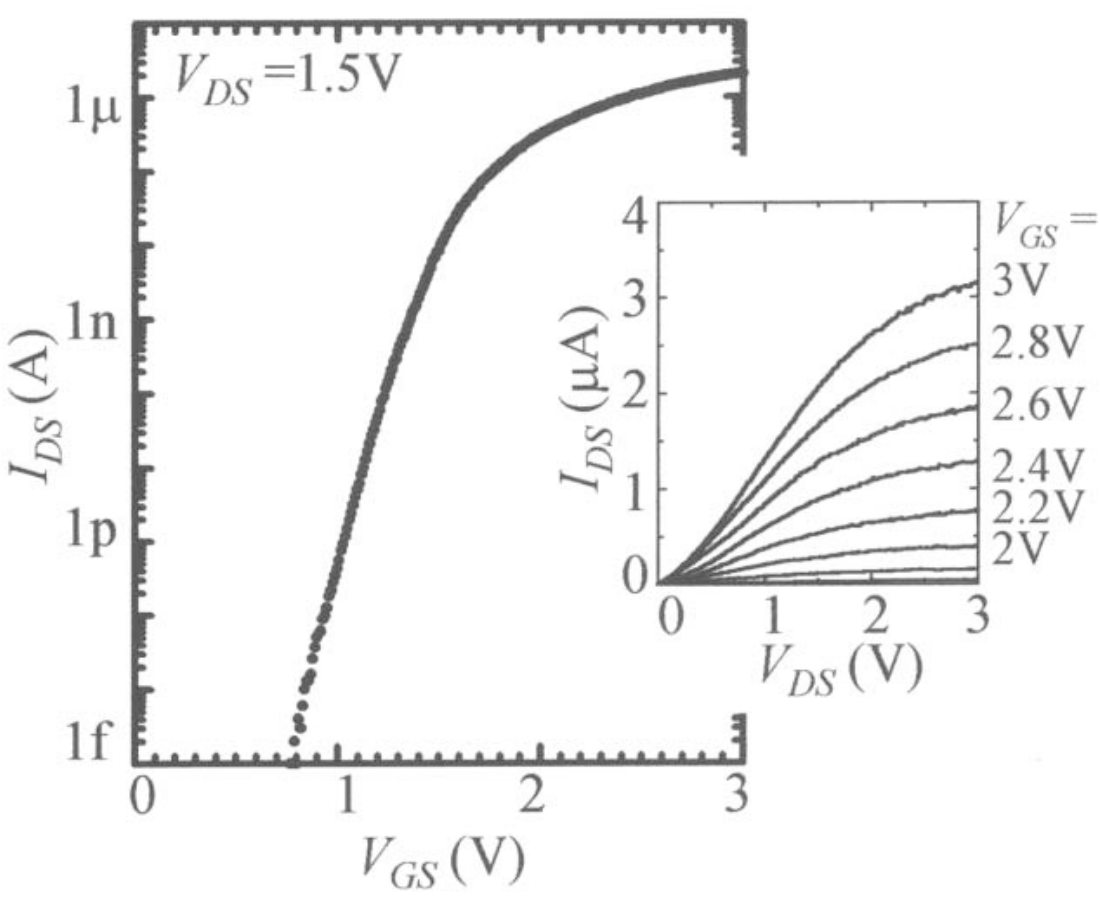

(b)

Figure 7.4.2: Fabricated PLEDTR. (a) Transmission electron micrograph, (b) measured drain current. 
silicon oxide data line

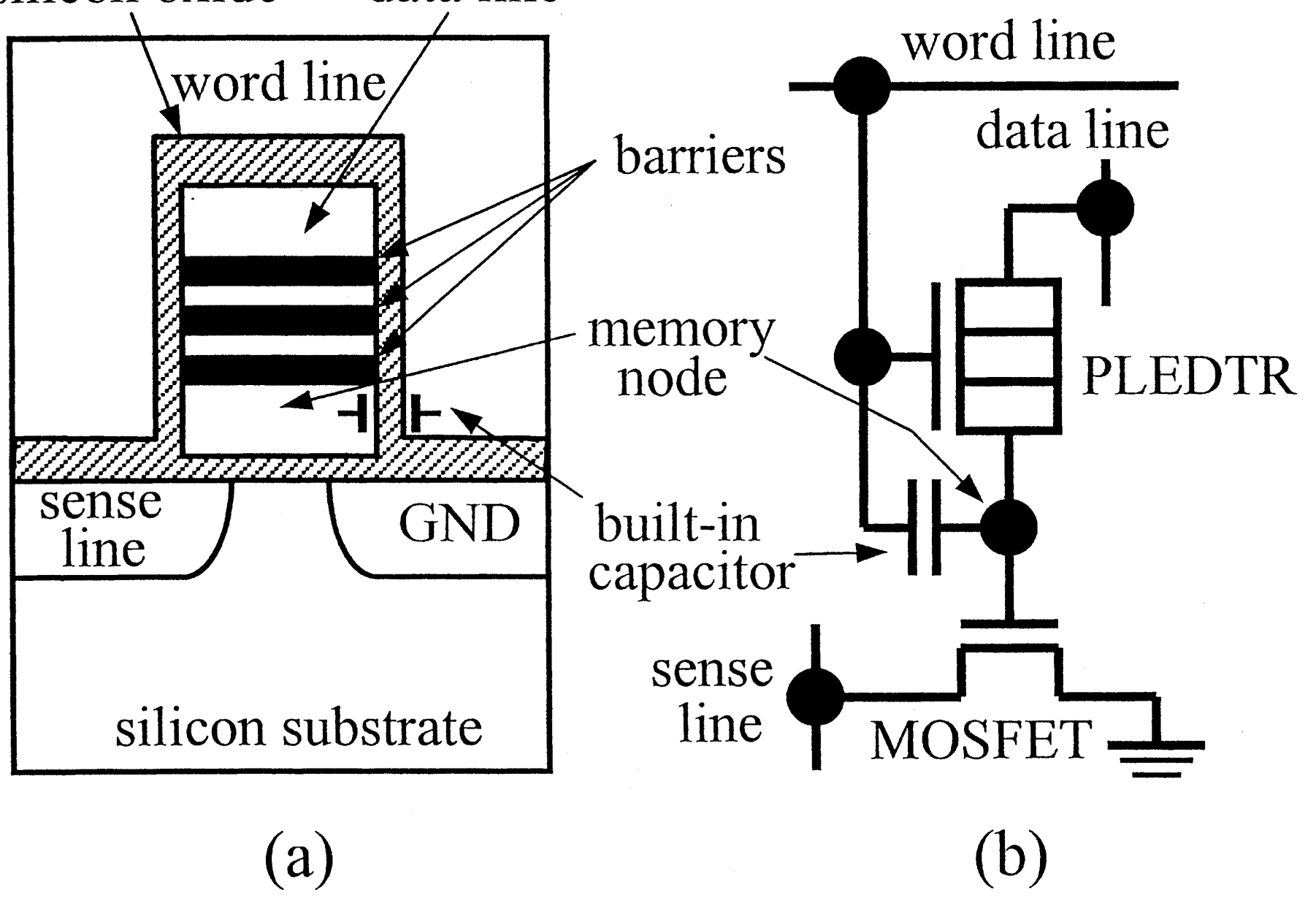

Figure 7.4.3: PLEDM cell (a) cross-section, (b) equivalent circuit diagram. 


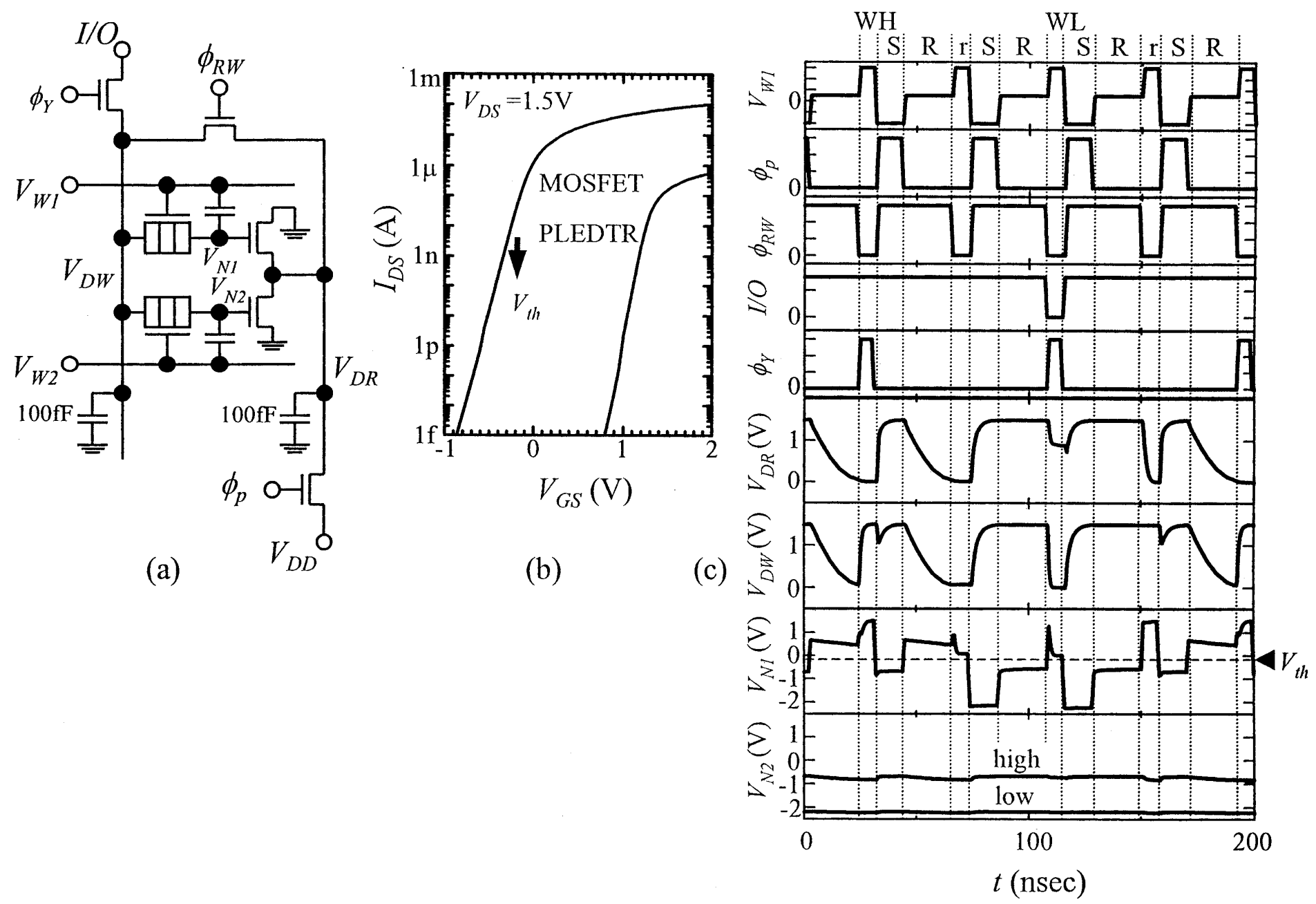

Figure 7.4.4: Simulation of memory cells.

(a) circuit diagram,

(b) transistor characteristics, (c) waveforms. 


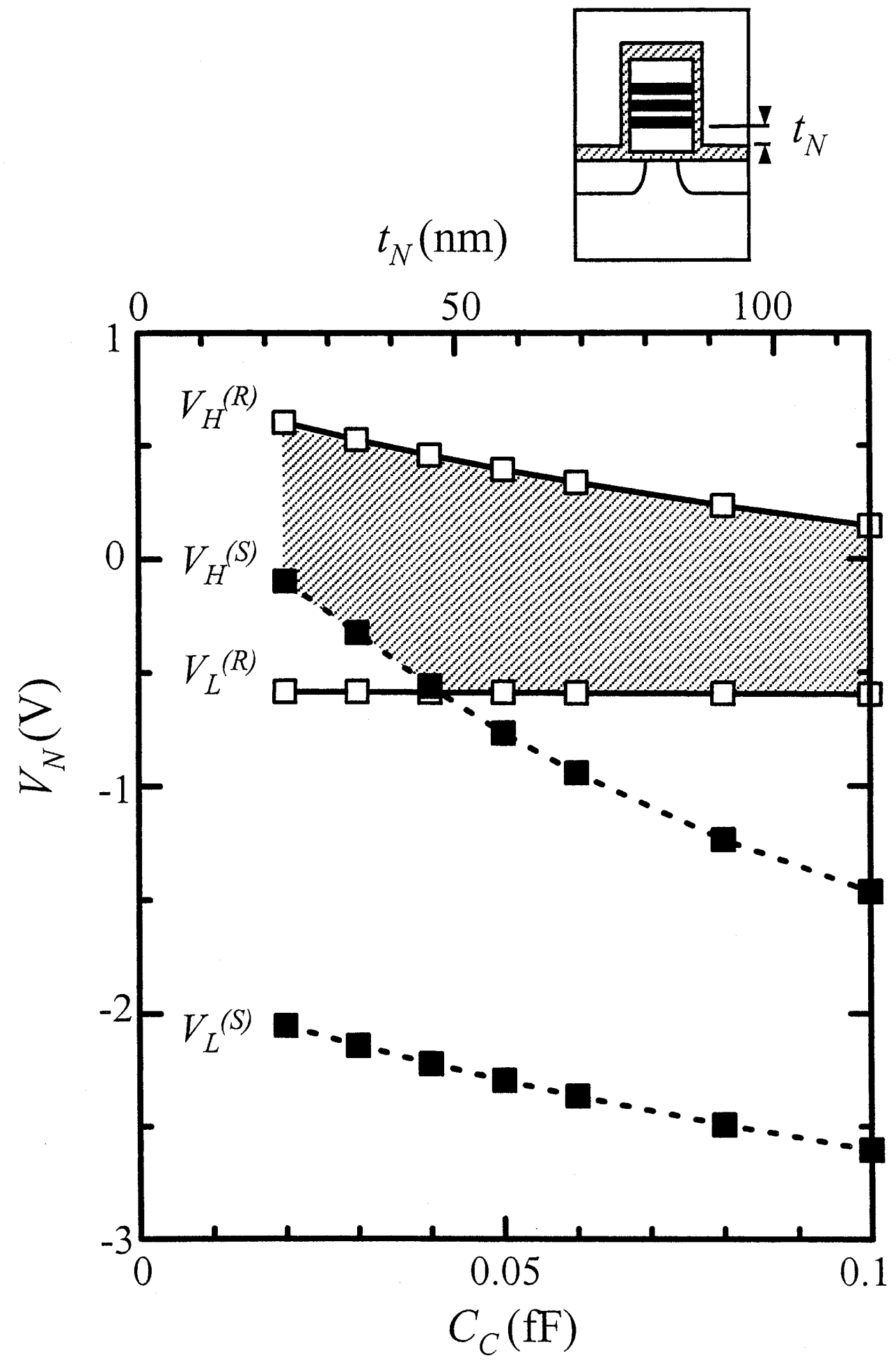

Figure 7.4.5: Simulated memory node voltages. 


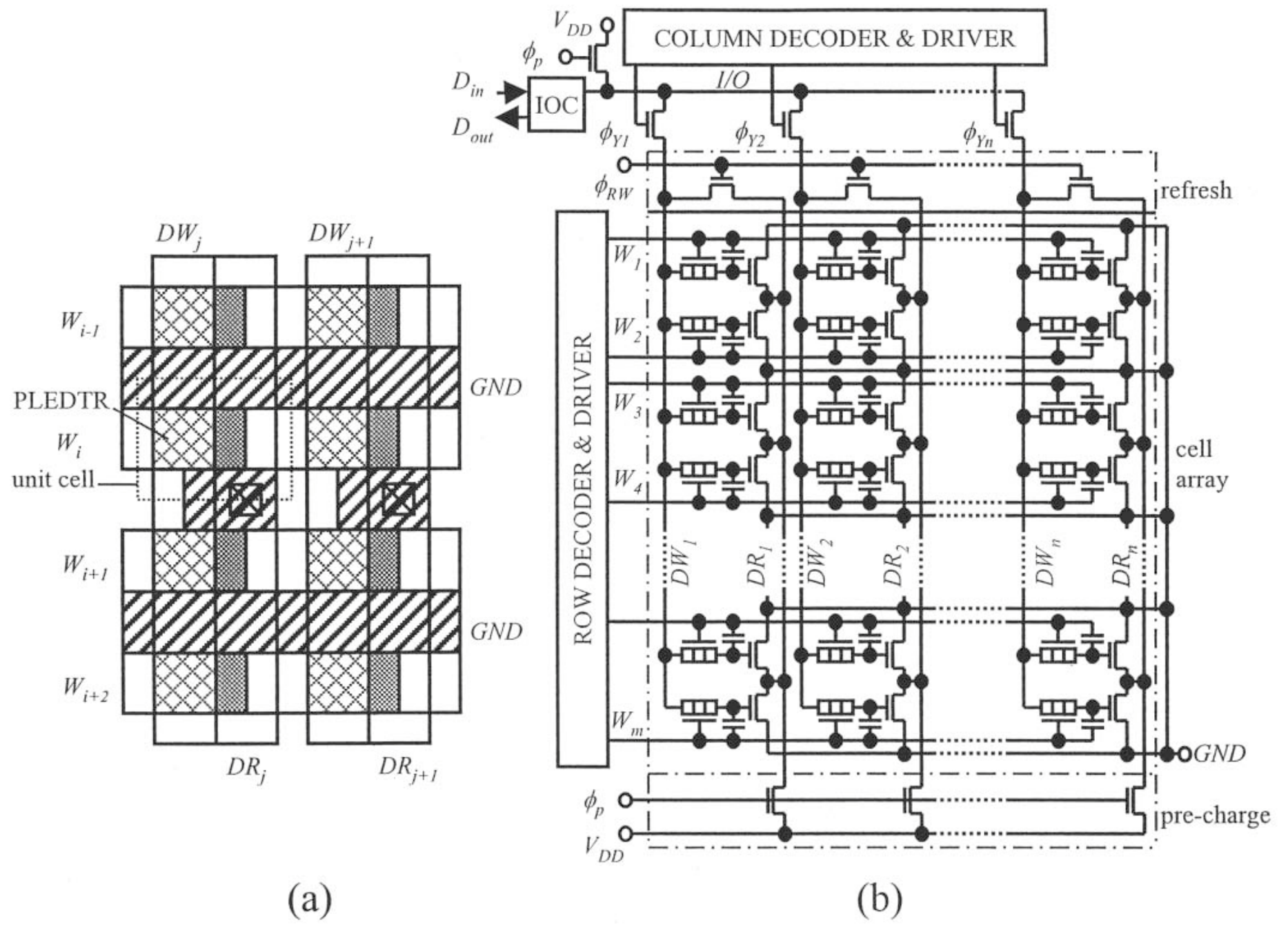

Figure 7.4.6: Memory device (a) cell layout, (b) schematic diagram. 